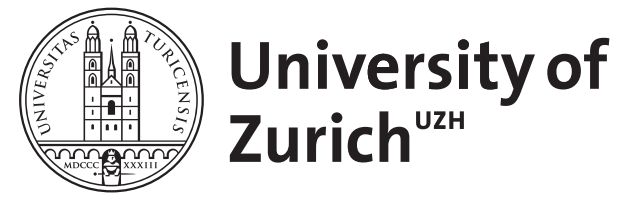

\title{
Magnetic field enhanced structural instability in EuTiO3
}

Guguchia, Z ; Keller, H ; Köhler, J ; Bussmann-Holder, A

DOI: https://doi.org/10.1088/0953-8984/24/49/492201

Posted at the Zurich Open Repository and Archive, University of Zurich ZORA URL: https://doi.org/10.5167/uzh-73428

Journal Article

Accepted Version

Originally published at:

Guguchia, Z; Keller, H; Köhler, J; Bussmann-Holder, A (2012). Magnetic field enhanced structural instability in EuTiO3. Journal of Physics: Condensed Matter, 24:492201.

DOI: https://doi.org/10.1088/0953-8984/24/49/492201 
preprint(July 20, 2012)

\title{
Magnetic field enhanced structural instability in $\mathrm{EuTiO}_{3}$
}

\author{
Z. Guguchia, ${ }^{1}$ H. Keller, ${ }^{1}$ J. Köhler ${ }^{2}$ and A. Bussmann-Holder ${ }^{2}$ \\ ${ }^{1}$ Physik-Institut der Universität Zürich, Winterthurerstrasse 190, CH-8057 Zürich, Switzerland \\ ${ }^{2}$ Max-Planck-Institut für Festkörperforschung, Heisenbergstr. 1, D-70569 Stuttgart, Germany
}

\begin{abstract}
$\mathrm{EuTiO}_{3}$ undergoes a structural phase transition from cubic to tetragonal at $T_{\mathrm{S}}=282 \mathrm{~K}$ which is not accompanied by any long range magnetic order. However, it is related to the oxygen ocathedra rotation driven by a zone boundary acoustic mode softening. Here we show that this displacive second order structural phase transition can be shifted to higher temperatures by the application of an external magnetic field $\left(\Delta T_{\mathrm{S}} \simeq 4 \mathrm{~K}\right.$ for $\left.\mu_{0} H=9 \mathrm{~T}\right)$. This observed field dependence is in agreement with theoretical predictions based on a coupled spin-anharmonic-phonon interaction model.
\end{abstract}

PACS numbers: 74.20.Mn, 74.25.Ha, 74.70.Xa, 76.75.+i

In the search for novel multiferroic materials the work of Katsufuji and Takagi [1] on $\mathrm{EuTiO}_{3}$ (ETO) has recently invoked hopes in having a new compound with the desired properties. They demonstrated that the dielectric constant of ETO increases steadily with decreasing temperature to show a dramatic decrease upon the onset of the antiferromagnetic phase transition at $T_{\mathrm{N}}=$ $5.5 \mathrm{~K}$. This unexpected behavior can be reversed by the application of a magnetic field and clearly demonstrates that a strong spin-lattice coupling is present in the system. However, a ferroelectric phase transition is absent, since not only quantum fluctuations suppress the complete softening of the ferroelectric soft mode [2,3], but also its energy is too large to find a finite extrapolated transition temperature as seen in $\mathrm{SrTiO}_{3}$ (STO) [4]. In spite of this negative result, ETO is an interesting candidate to search for novel spin-lattice interaction effects, since opposite to other multiferroics, not the transition metal d-states are occupied and responsible for the low temperature magnetic properties, but the $A$-site ion in $A B \mathrm{O}_{3}$ accounts for the antiferromagnetic phase [1]. The close analogy between ETO and STO has recently been shown [5] to be complemented by the fact that both compounds undergo a structural phase transition from cubic to tetragonal. While the one of STO takes place at $T_{\mathrm{S}}=$ $105 \mathrm{~K}$ [4, 6-12], the corresponding one of ETO occurs at much higher temperatures, namely at $T_{\mathrm{S}}=282 \mathrm{~K}$, where the space group of the structure changes from $P m \overline{3} m$ to $I 4 / m c m[13,14]$. This phase transition has been explored by various techniques: specific heat measurements, electron paramagnetic resonance (EPR), muon-spin rotation $(\mu \mathrm{SR})$, and X-ray scattering $[5,13,15,16]$. All experimental results clearly evidence the structural instability, and it was also detected in the mixed crystals of $\mathrm{Sr}_{1-x} \mathrm{Eu}_{x} \mathrm{TiO}_{3}$ [15].

An especially interesting result was obtained from $\mu \mathrm{SR}$ experiments where a finite relaxation rate $\lambda_{\text {para }}$ could be detected in the paramagnetic phase of ETO up to elevated temperatures even exceeding $T_{\mathrm{S}}$ [16]. Its temperature dependence follows closely the one of the soft zone boundary mode as well as the one of the inverse EPR line width. This finding not only proves that the spin-lattice coupling is strong, but also demonstrates that correlated fluctuating spins are present which form finite size clusters. Theoretically, this observation has been modeled within a spin-lattice coupled model Hamiltonian from which a hybrid paramagnon phonon coupled mode has been predicted to appear far above $T_{\mathrm{N}}[5,15,16]$. Another consequence of this approach is that a magnetic field has a substantial influence on the eigenfrequencies of the system. This is shown in Fig. 1a where the three frequency branches, optic, acoustic and paramagnon, are shown for $T=300 \mathrm{~K}$, as a function of the spin-lattice coupling $\varepsilon$ which is proportional to the magnetic field $H$ [17]. With increasing coupling the zone boundary transverse acoustic (TA) mode frequency decreases systematically whereas the transverse optic (TO) long wave length mode is not affected by the magnetic field. There is, however, a strong paramagnon-optic-phonon coupling at finite momentum $\mathrm{q} \simeq 0.25$ which suggests the formation of lattice mediated spin cluster formation.

The influence of the magnetic field on the acoustic mode is not only accompanied by its softening at the zone boundary, but substantial anomalies are also seen in the long wave length limit, pointing to a pronounced softening of the elastic constants under an applied magnetic field, namely strong magneto-elastic coupling which is shown in Fig. 1b where the acoustic mode dispersion is normalized to its zero field dispersion. For small field strengths this coupling is rather inefficient, but becomes distinct with increasing field.

The softening of the zone boundary mode frequency with increasing field is an indirect proof that the structural phase transition temperature $T_{\mathrm{S}}$ is also field dependent. This has been tested by performing specific heat measurements in an applied magnetic field over a broad temperature regime including the Néel phase.

The sample preparation has been described in Ref. 5 . The specific heat measurements (relaxor type calorimeter Physical Properties Measurements System Quantum Design) were first performed in zero field and then repeated in fields of 3,7 , and $9 \mathrm{~T}$. The results are shown 

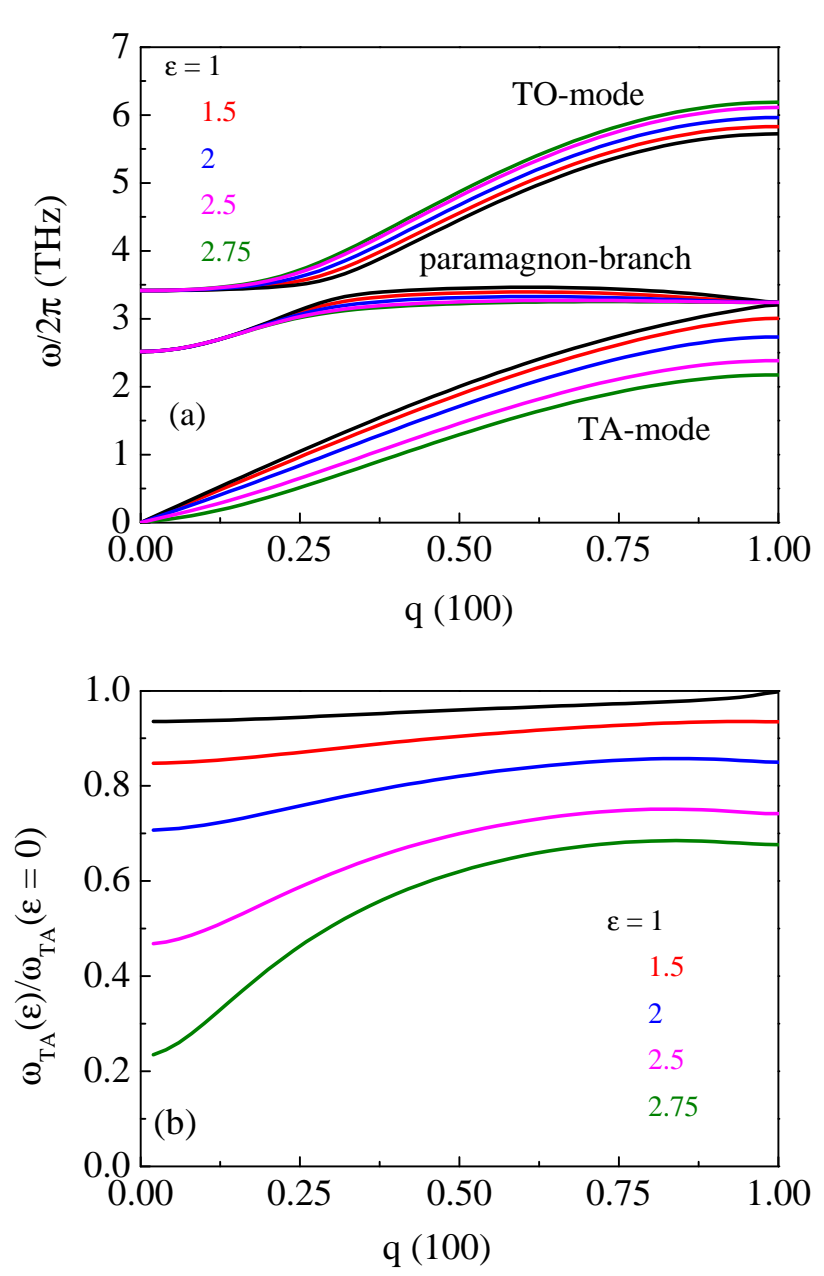

FIG. 1: (Color online) a) Dispersion of the acoustic, optic and paramagnon modes at $T=300 \mathrm{~K}$ for different coupling strengths $\varepsilon \propto H$ as indicated in the figure. b) Acoustic mode dispersion normalized to the zero field dispersion for the same values of $\varepsilon$ as in the main part of the figure.

in Fig. 2. Importantly, the zero field data (Fig. 2a) are in excellent agreement with our previously reported data [5]. For the field dependent data it is obvious from Figs. $2 \mathrm{a}$ and $2 \mathrm{~b}$ that the low temperature specific heat anomaly caused by the antiferromagnetic phase transition diminishes with increasing field in accordance with the dielectric data [1] and field dependent specific heat data [18]. At high temperatures the anomaly stemming from the structural phase transition is visible as a distinct peak which is in the focus of the following discussion (Fig. 2a). By concentrating on a limited temperature range around $T_{\mathrm{S}}$ the anomaly is enlarged and better visible as shown in Fig. 3a. Obviously a shift of $T_{\mathrm{S}}$ to higher temperatures takes place with increasing $H$ which is shown in Fig. 3b. While the data of $0 \mathrm{~T}$ and $3 \mathrm{~T}$ are almost identical, a nonlinear enhancement of $T_{\mathrm{S}}$ is observed for larger magnetic fields. While it is well known, though rather rarely observed, that structural phase transitions
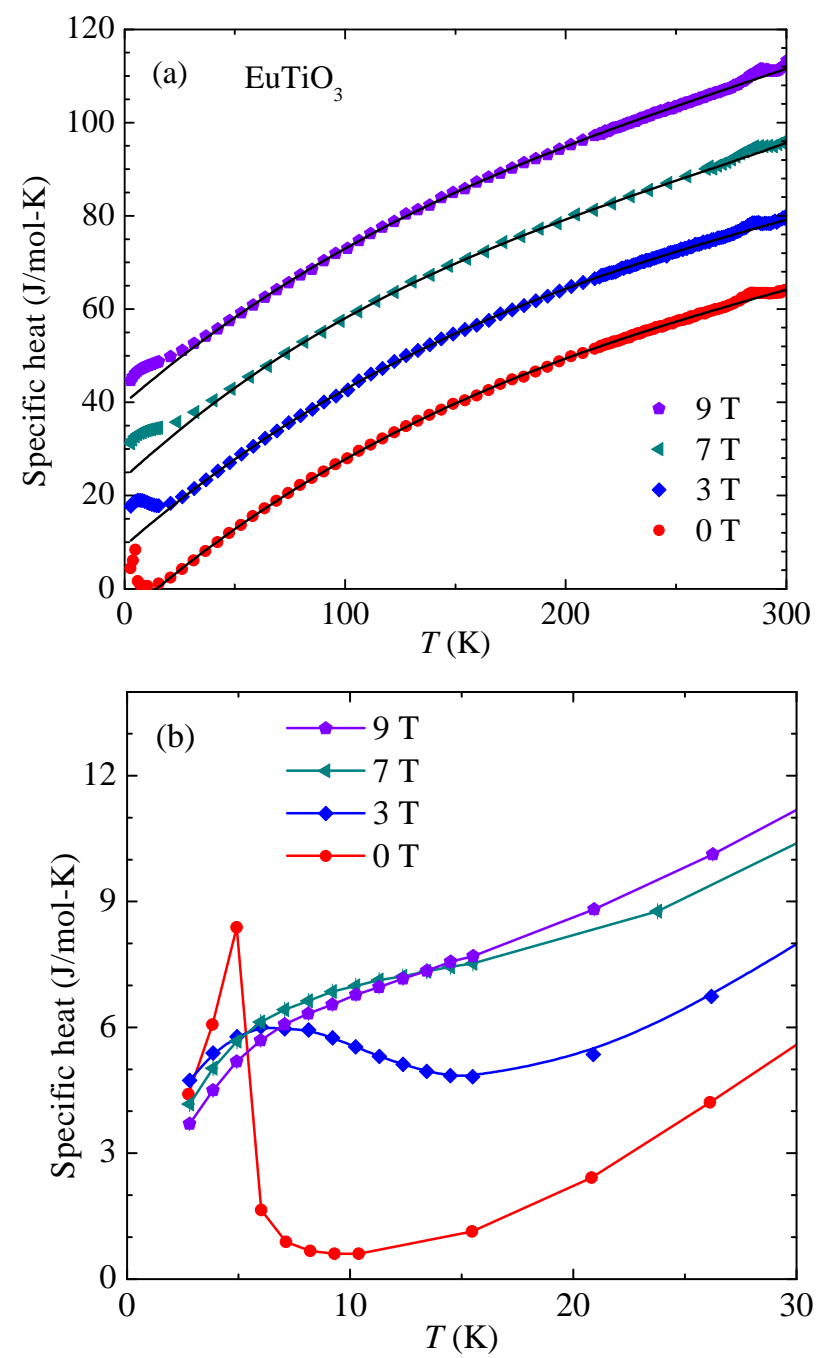

FIG. 2: (Color online) a) Specific heat of ETO as a function of temperature at $\mu_{0} H=0 \mathrm{~T}, 3 \mathrm{~T}, 7 \mathrm{~T}$, and $9 \mathrm{~T}$. For clarity the data are shifted by $15 \mathrm{~J} / \mathrm{mol}-\mathrm{K}$ relative to each other with increasing magnetic field. b) Specific heat of ETO at low temperatures showing the anomaly at $T_{\mathrm{N}}=5.5 \mathrm{~K}$ (same data as in Fig. 2a).

involving a transition to a magnetically ordered phase can be tuned by a magnetic field, the field dependence of the purely structural transition of ETO without involvement of magnetic ordering is new. It has also no analogies to other incipient ferroelectric perovskites, where the oxygen octahedral rotation instability is insensitive to a magnetic field. The consequences are multifold since a tuning of piezoelectric and pyroelectric effects in amorphous ETO analogous to STO and $\mathrm{BaZrO}_{3}[19,20]$ is possible by a magnetic field and novel functionalities can be expected. The experimental data can be compared to the theoretically derived ones by plotting the zone boundary acoustic mode as a function of $\varepsilon$ (Fig. 4). As is obvious from Fig. 4 a nonlinear dependence of $\omega_{\mathrm{TA}}^{2}(q$ $=2 \pi / a)$ on $\varepsilon$ is obtained with an analogous dependence 

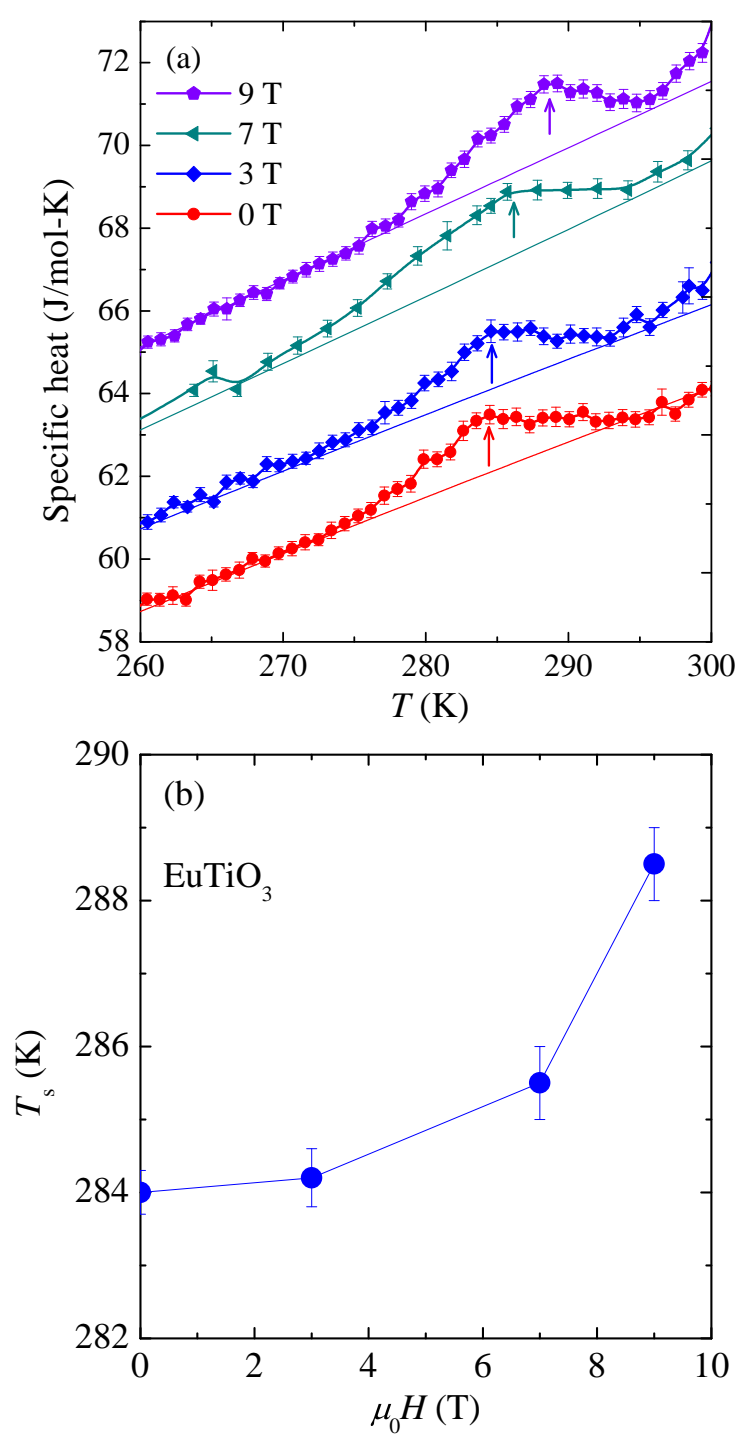

FIG. 3: (Color online) a) The specific heat of ETO within a limited temperature regime around the structural phase transition temperature $T_{\mathrm{S}}$ for fields of $0,3,7,9 \mathrm{~T}$. For clarity the data are shifted by $2 \mathrm{~J} / \mathrm{mol}-\mathrm{K}$ relative to each other with increasing magnetic field. b) $T_{\mathrm{S}}$ as a function of $H$ as derived from Fig. 3a.

of the frequency on the coupling as $T_{\mathrm{S}}$ on $H$ (Fig. 3b). Importantly, the zone boundary acoustic mode decreases with increasing field indicating the increased tendency towards the structural instability in accordance with the experiment (Figs. 3a and 3b). The observed magnetic field dependence of the structural instability cannot be explained by the fact that the phase transition is decoupled from the Eu spins which do not show any long range order in this temperature regime. Instead and in accordance with our previous data and analysis [16], it must be concluded that finite size spin ordered clusters exist in ETO at high temperatures which strongly interact with the lattice dynamics. Since it is well known for STO that

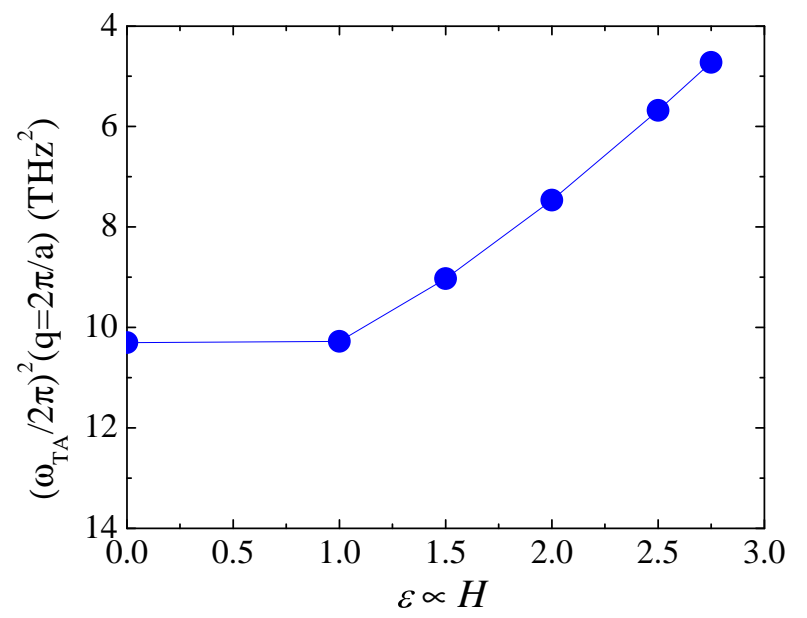

FIG. 4: (Color online) Field dependence of the acoustic zone boundary mode frequency $\omega_{\mathrm{TA}}^{2}(q=2 \pi / a)$ as derived from Fig. 1.

dynamical precursor effects are present above the structural phase transition temperature which increase in size upon approaching $T_{\mathrm{S}}[21]$ we can assume also for ETO that similar dynamics are realized here as well. However, these finite size clusters obviously couple to the spin subsystem and induce local spin ordering. The magnetic field effect contributes to their spatial extent and influences thereby the growth of the precursor dynamics. As such a novel and interesting interplay between the lattice and the spins takes place which to our knowledge has not been reported before. Another consequence of our combined experimental - theoretical approach is the observation that the magnetic field has pronounced effects on the elastic properties of ETO, namely a strong elastic softening. This is in contrast to temperature effects where no such softening is present [22], suggesting that ETO should exhibit a huge magneto-elastic coupling effect leading to novel functionalities.

In conclusion, we have shown that the structural phase transition of ETO can be manipulated by an external magnetic field which shifts $T_{\mathrm{S}}$ to higher temperatures with the dependence of $T_{\mathrm{S}}$ on $H$ being nonlinear. Theoretically a similar dependence of the zone boundary transverse mode frequency on the spin-lattice coupling is found where an increased softening with increasing coupling strength $\varepsilon$ takes place. The additional implications are large magneto-elastic interactions which should be observable by resonant ultrasound spectroscopy. The experimental data together with their interpretation suggest that dynamical precursor clusters form above the actual structural phase transition temperature which are tied to the Eu spins to induce a locally ordered spin alignment via the bridging oxygen ions thus possibly leading to ferromagnetic domains through the superexchange mechanism [21]. 


\section{ACKNOWLEDGMENTS}

This work was supported by the Swiss National Science Foundation and the SCOPES grant No.IZ73Z0_128242.

[1] T. Katsufuji and H. Takagi, Phys. Rev. B 64, 054415 (2001).

[2] S. Kamba, D. Nuzhnyy, P. Vaněk, M. Savinov, K. Knižek, Z. Shen, E. Santavá, K. Maca, M. Sadowski, and J. Petzelt, Europhys. Lett. 80, 27002 (2007).

[3] V. Goian, S. Kamba, J. Hlinka, P. Vaněk, A. A. Balik, T. Kolodiazhnyi, and J. Petzelt, Europ. Phys. J. B 71, 429 (2009).

[4] K.A. Müller, and H. Burkhard, Phys. Rev. B 19, 3593 (1979).

[5] A. Bussmann-Holder, J. Köhler, R. K. Kremer, and J. M. Law, Phys. Rev. B 83, 212102 (2011).

[6] K.A. Müller, Phys. Rev. Lett. 2, 341 (1959).

[7] P.A. Fleury, J. F. Scott, and J. M. Worlock, Phys. Rev. Lett. 21, 16 (1968).

[8] J.S. Kim, M. Itoh, and T. Nakamura, J. Solid State Chem. 101, 77 (1992).

[9] H. Unoki and T. Sakudo, J. Phys. Soc. Jpn. 23, 546 (1967).

[10] E.J. Kirkpatrick, K.A. Müller, and R.S. Rubins, Phys.
Rev. A 135, 86 (1964).

[11] G. Shirane and Y. Yamada, Phys. Rev. 177, 858 (1969).

[12] R.A. Cowley, W.J.L. Buyers, and G. Dolling, Solid State Commun. 7, 181 (1969).

[13] J. Köhler, R. Dinnebier, and A. Bussmann-Holder, Phase Transitions, in press; arXiv:1205.5374.

[14] M. Allieta, M. Scavini, L.J. Spalek, V. Scagnoli, H.C. Walker, C. Panagopoulos, S.S. Saxena, T. Katsufuji, and C. Mazzoli, Phys. Rev. B 85, 184107 (2012).

[15] Z. Guguchia, A. Shengelaya, H. Keller, J. Köhler, and A. Bussmann-Holder, Phys. Rev. B 85, 134113 (2012).

[16] A. Bussmann-Holder, Z. Guguchia, J. Köhler, H. Keller, A. Shengelaya, and A.R. Bishop, arXiv:1205.6287v1.

[17] E.H. Jacobsen and K.W.H. Stevens, Phys. Rev. 129, 2036 (1963).

[18] A.P. Petrović, Y. Kato, S.S. Sunku, T. Ito, P. Sengupta, L. Spalek, M. Shimuta, T. Katsufuji, C.D. Batista, S. Saxena, and C. Panagopoulos, arXiv:1204.0150v1.

[19] D. Ehre, V. Lyahovitskaya, A. Tagantsev, I. Lubomirsky, Adv. Mater. 19, 1515 (2007).

[20] A.I. Frenkel, D. Ehre, V. Lyahovitskaya, L. Kanner, E. Wachtel, and I. Lubomirsky, Phys. Rev. Lett. 99, 215502 (2007).

[21] K. Roleder, A. Bussmann-Holder, M. Górny, K. Szot, and A. M. Glazer, Phase Transitions 1-10, iFirst (2012).

[22] J.L. Bettis, M.-H. Whangbo, J. Köhler, A. BussmannHolder, and A.R. Bishop, Phys. Rev. B 84, 184114 (2011). 